\title{
DESCENDENTS ON LOCAL CURVES: STATIONARY THEORY
}

\author{
R. PANDHARIPANDE AND A. PIXTON
}

\begin{abstract}
The stable pairs theory of local curves in 3-folds (equivariant with respect to the scaling 2 -torus) is studied with stationary descendent insertions. Reduction rules are found to lower descendents when higher than the degree. Factorization then yields a simple proof of rationality in the stationary case and a proof of the functional equation related to inverting $q$. The method yields an effective determination of stationary descendent integrals. The series $\mathbf{Z}_{d,(d)}^{\text {cap }}\left(\tau_{d}(\mathrm{p})\right)$ plays a special role and is calculated exactly using the stable pairs vertex and an analysis of the solution of the quantum differential equation for the Hilbert scheme of points of the plane.
\end{abstract}

\section{Contents}

$0 . \quad$ Introduction . . . . . . . . . . . . . . . 1

1. Reduction for stationary descendents . . . . . . . . . 6

2. Factorization and rationality . . . . . . . . . . . . . . 9 9 9

3. Localization formalism . . . . . . . . . . . . . . . 12

4. Calculation of $Z_{d,(d)}^{\text {cap }}\left(\tau_{d}(\mathrm{p})\right)^{\mathbf{T}} \ldots \ldots \ldots \ldots \ldots \ldots$

References ...................... 25

\section{INTRODUCTION}

0.1. Relative local curves. The geometry of a 3-fold local curve consists of a split rank 2 bundle $N$ on a nonsingular projective curve $C$ of genus $g$,

$$
N=L_{1} \oplus L_{2} .
$$

The splitting determines a scaling action of a 2-dimensional torus

$$
T=\mathbb{C}^{*} \times \mathbb{C}^{*}
$$

Date: September 2011. 
on $N$. The level of the splitting is the pair of integers $\left(k_{1}, k_{2}\right)$ where,

$$
k_{i}=\operatorname{deg}\left(L_{i}\right) .
$$

Of course, the scaling action and the level depend upon the choice of splitting (11).

The fiber of $N$ over a point $p \in C$ determines a $T$-invariant divisor

$$
N_{p} \subset N
$$

isomorphic to $\mathbb{C}^{2}$ with the standard $T$-action. We will consider the local stable pairs theory of $N$ relative to the divisor

$$
S=\bigcup_{i=1}^{r} N_{p_{i}} \subset N
$$

determined by the fibers over $p_{1}, \ldots, p_{r} \in C$. Let $P_{n}(N / S, d)$ denote the relative moduli space of stable pair\$1, see [22].

For each $p_{i}$, let $\eta^{i}$ be a partition of $d$ weighted by the equivariant Chow ring,

$$
A_{T}^{*}\left(N_{p_{i}}, \mathbb{Q}\right) \stackrel{\simeq}{=}\left[s_{1}, s_{2}\right],
$$

of the fiber $N_{p_{i}}$. By Nakajima's construction, a weighted partition $\eta^{i}$ determines a $T$-equivariant class

$$
\mathrm{C}_{\eta^{i}} \in A_{T}^{*}\left(\operatorname{Hilb}\left(N_{p_{i}}, d\right), \mathbb{Q}\right)
$$

in the Chow ring of the Hilbert scheme of points. In the theory of stable pairs, the weighted partition $\eta^{i}$ specifies relative conditions via the boundary map

$$
\epsilon_{i}: P_{n}(N / S, d) \rightarrow \operatorname{Hilb}\left(N_{p_{i}}, d\right)
$$

An element $\eta \in \mathcal{P}(d)$ of the set of partitions of $d$ may be viewed as a weighted partition with all weights set to the identity class

$$
1 \in A_{T}^{*}\left(N_{p_{i}}, \mathbb{Q}\right) .
$$

The Nakajima basis of $A_{T}^{*}\left(\operatorname{Hilb}\left(N_{p_{i}}, d\right), \mathbb{Q}\right)$ consists of identity weighted partitions indexed by $\mathcal{P}(d)$.

Let $s_{1}, s_{2} \in H_{\mathbf{T}}^{*}(\bullet)$ be the first Chern classes of the standard representations of the first and second $\mathbb{C}^{*}$-factors of $T$ respectively. The $T$-equivariant intersection pairing in the Nakajima basis is

$$
g_{\mu \nu}=\int_{\operatorname{Hilb}\left(N_{p_{i}}, d\right)} \mathrm{C}_{\mu} \cup \mathrm{C}_{\nu}=\frac{1}{\left(s_{1} s_{2}\right)^{\ell(\mu)}} \frac{(-1)^{d-\ell(\mu)}}{\mathfrak{z}(\mu)} \delta_{\mu, \nu},
$$

\footnotetext{
${ }^{1}$ The curve class is $d$ times the zero section $C \subset N$.
} 
DESCENDENTS ON LOCAL CURVES: STATIONARY THEORY 3

where

$$
\mathfrak{z}(\mu)=\prod_{i=1}^{\ell(\mu)} \mu_{i} \cdot|\operatorname{Aut}(\mu)|
$$

Let $g^{\mu \nu}$ be the inverse matrix.

0.2. Descendents. We define descendents in the relative stable pairs theory of local curves by the slant products with universal sheaf following [20].

There exists a universal sheaf on the universal 3 -fold $\mathcal{N}$ over the moduli space $P_{n}(N / S, d)$,

$$
\mathbb{F} \rightarrow \mathcal{N}
$$

For a stable pair $[\mathcal{O} \rightarrow F] \in P_{n}(N / S, d)$, the restriction of $\mathbb{F}$ to the fiber

$$
\mathcal{N}_{[\mathcal{O} \rightarrow F]} \subset \mathcal{N}
$$

is canonically isomorphic to $F$. Let

$$
\begin{gathered}
\pi_{N}: \mathcal{N} \rightarrow N, \\
\pi_{P}: \mathcal{N} \rightarrow P_{n}(N / S, d)
\end{gathered}
$$

be the canonical projections.

By the stability conditions for the relative theory of stable pairs, $\mathbb{F}$ has a finite resolution by locally free sheaves. Hence, the Chern character of the universal sheaf $\mathbb{F}$ is well-defined. By definition, the operation

$$
\pi_{P *}\left(\pi_{N}^{*}(\gamma) \cdot \operatorname{ch}_{2+i}(\mathbb{F}) \cap\left(\pi_{P}^{*}(\cdot)\right): H_{*}\left(P_{n}(N / S, d)\right) \rightarrow H_{*}\left(P_{n}(N / S, d)\right)\right.
$$

is the action of the descendent $\tau_{i}(\gamma)$, where $\gamma \in H^{*}(C, \mathbb{Z})$. The pushforwards are defined by $T$-equivariant residues as in [3, 18].

We will use bracket notation for descendents,

$$
\left\langle\prod_{j=1}^{\ell} \tau_{i_{j}}\left(\gamma_{j}\right)\right\rangle_{n, d}^{N, \eta^{1}, \ldots, \eta^{r}}=\int_{\left.\left[P_{n}(N / S, d)\right]\right]^{v i r}} \prod_{j=1}^{\ell} \tau_{i_{j}}\left(\gamma_{j}\right) \prod_{i=1}^{r} \epsilon_{i}^{*}\left(\mathrm{C}_{\eta^{i}}\right) .
$$

The partition function is denoted by

$$
\mathrm{Z}_{d, \eta^{1}, \ldots, \eta^{r}}^{N / S}\left(\prod_{j=1}^{\ell} \tau_{i_{j}}\left(\gamma_{j}\right)\right)^{T}=\sum_{n}\left\langle\prod_{j=1}^{\ell} \tau_{i_{j}}\left(\gamma_{j}\right)\right\rangle_{n, d}^{N, \eta^{1}, \ldots, \eta^{r}} q^{n} .
$$

The following basic result is proved in [20].

Theorem. $\mathbf{Z}_{d, \eta^{1}, \ldots, \eta^{r}}^{N / S}\left(\prod_{j=1}^{k} \tau_{i_{j}}\left(\gamma_{j}\right)\right)^{T}$ is the Laurent expansion in $q$ of a rational function in $\mathbb{Q}\left(q, s_{1}, s_{2}\right)$. 
0.3. Stationary theory. Our main results here concern stationary descendents in the stable pairs theory of local curves. Let

$$
\mathrm{p} \in H^{2}(C, \mathbb{Z})
$$

be the class of a point. The stationary descendents are $\tau_{k}(\mathrm{p})$. The methods of the paper, while not fully applicable to other descendents, are much simpler and more effective than the techniques of [20, 21].

Our first result concerns reduction rules for stationary descendents in the theory of local curves.

Theorem 1. For $k>d$, there exist universal polynomials

$$
f_{k, d}\left(x_{1}, \ldots, x_{d}\right) \in \mathbb{Q}\left(s_{1}, s_{2}\right)\left[x_{1}, \ldots, x_{d}\right]
$$

for which the degree $d$ descendent theory of local curves satisfies the reduction rule

$$
\tau_{k}(\mathrm{p}) \mapsto f_{k, d}\left(\tau_{1}(\mathrm{p}), \ldots, \tau_{d}(\mathrm{p})\right)
$$

Explicitly, Theorem 1 yields the following equality for $T$-equivariant integrals:

$$
\begin{aligned}
\mathrm{Z}_{d, \eta^{1}, \ldots, \eta^{r}}^{N / S}\left(\tau_{k}(\mathrm{p}) \cdot \prod_{j=1}^{\ell} \tau_{i_{j}}\left(\gamma_{j}\right)\right)^{T} & = \\
& \mathrm{Z}_{d, \eta^{1}, \ldots, \eta^{r}}^{N / S}\left(f_{k, d}\left(\tau_{1}(\mathrm{p}), \ldots \tau_{d}(\mathrm{p})\right) \cdot \prod_{j=1}^{\ell} \tau_{i_{j}}\left(\gamma_{j}\right)\right)^{T}
\end{aligned}
$$

for $k>d$ and all $\gamma_{j} \in H^{*}(C, \mathbb{Z})$. Theorem 1 is proven in Section 1 .

Via Theorem 1, factorization properties of the relative conditions, and the established rationality of the stable pairs theory of local curves without insertions, we obtain our second result in Section 2.

Theorem 2. The stationary series $\mathbf{Z}_{d, \eta^{1}, \ldots, \eta^{r}}^{N / S}\left(\prod_{j=1}^{k} \tau_{i_{j}}(\mathbf{p})\right)^{T}$ is the Laurent expansion in $q$ of a rational function $F\left(q, s_{1}, s_{2}\right) \in \mathbb{Q}\left(q, s_{1}, s_{2}\right)$ satisfying the functional equation

$$
F\left(q^{-1}, s_{1}, s_{2}\right)=(-1)^{\Delta+|\eta|-\ell(\eta)+\sum_{j=1}^{k} i_{j}} q^{-\Delta} F\left(q, s_{1}, s_{2}\right),
$$

where the constants are defined by

$$
\Delta=\int_{\beta} c_{1}\left(T_{N}\right), \quad|\eta|=\sum_{i=1}^{r}\left|\eta^{i}\right|, \quad \text { and } \quad \ell(\eta)=\sum_{i=1}^{r} \ell\left(\eta^{i}\right) .
$$

Here, $T_{N}$ is the tangent bundle of the 3 -fold $N$, and $\beta$ is the curve class given by $d$ times the 0 -section. Our proof of Theorem 2 is much easier than the rationality results of [20]. Moreover, we do not know how to derive the functional equation from the methods of [20]. 
DESCENDENTS ON LOCAL CURVES: STATIONARY THEORY 5

As a step in the proof of Theorem 2, we show the entire stationary descendent theory is determined from the theory of local curves without insertions and the set of series

$$
\mathrm{Z}_{d,(d)}^{\mathrm{cap}}\left(\tau_{d}(\mathrm{p})\right)^{T}=\sum_{n}\left\langle\tau_{d}(\mathrm{p})\right\rangle_{n, d}^{N,(d)} q^{n}, \quad d>0 .
$$

Here, the cap geometry is $\mathbb{P}^{1}$ relative to $\infty \in \mathbb{P}^{1}$. A central result of the paper is the following calculation.

Theorem 3. We have

$$
\mathrm{Z}_{d,(d)}^{\mathrm{cap}}\left(\tau_{d}(\mathrm{p})\right)^{T}=\frac{q^{d}}{d !}\left(\frac{s_{1}+s_{2}}{s_{1} s_{2}}\right) \frac{1}{2} \sum_{i=1}^{d} \frac{1+(-q)^{i}}{1-(-q)^{i}}
$$

In the above formula, the coefficient of $q^{d}$,

$$
\left\langle\tau_{d},(d)\right\rangle_{\operatorname{Hilb}\left(\mathbb{C}^{2}, d\right)}=\frac{1}{2 \cdot(d-1) !}\left(\frac{s_{1}+s_{2}}{s_{1} s_{2}}\right),
$$

is the classical $T$-equivariant pairing on the Hilbert scheme of $d$ points on $\mathbb{C}^{2}$. The proof of Theorem 3 is given in Section 4.

Very few exact calculations for descendents in 3-fold sheaf theories have previously been found. Theorem 3 provides a closed form for the most fundamental descendent series in the stationary theory of local curves. The derivation uses the localization methods of [23] together with an analysis of the fundamental solution of the quantum differential equation of the Hilbert scheme of points of the plane.

The descendent partition functions for the stable pairs theory of local curves have very restricted denominators when considered as rational functions in $q$ with coefficients in $\mathbb{Q}\left(s_{1}, s_{2}\right)$. A basic result proven in Section 9 of [20] is the following.

Theorem. The denominators of the degree $d$ descendent partition functions $\mathrm{Z}_{d, \eta^{1}, \ldots, \eta^{r}}^{N / S}\left(\prod_{j=1}^{k} \tau_{i_{j}}(\mathrm{p})\right)^{T}$ are products of factors of the form $q^{s}$ and

$$
1-(-q)^{r}
$$

for $1 \leq r \leq d$.

Certainly the calculation of Theorem 3 is consistent with the denominator result.

0.4. Acknowledgements. We thank J. Bryan, D. Maulik, A. Oblomkov, A. Okounkov, and R. Thomas for several discussions about stable pairs, descendents, and the quantum cohomology of the Hilbert scheme of points of the plane. V. Shende's questions at the Newton Institute 
about the $q \leftrightarrow q^{-1}$ symmetry for descendents prompted us to work out the proof of the functional equation.

R.P. was partially supported by NSF grant DMS-0500187 and DMS1001154. A.P. was supported by a NDSEG graduate fellowship. The paper was completed while visiting the Instituto Superior Técnico in Lisbon where R.P. was supported by a Marie Curie fellowship and a grant from the Gulbenkian foundation.

\section{REDUCTION FOR STATIONARY DESCENDENTS}

1.1. Cap geometry. The capped 1-leg geometry concerns the trivial bundle,

$$
N=\mathcal{O}_{\mathbb{P}^{1}} \oplus \mathcal{O}_{\mathbb{P}^{1}} \rightarrow \mathbb{P}^{1}
$$

relative to the fiber

$$
N_{\infty} \subset N
$$

over $\infty \in \mathbb{P}^{1}$. The total space $N$ naturally carries an action of a 3 dimensional torus

$$
\mathbf{T}=T \times \mathbb{C}^{*} .
$$

Here, $T$ acts as before by scaling the factors of $N$ and preserving the relative divisor $N_{\infty}$. The $\mathbb{C}^{*}$-action on the base $\mathbb{P}^{1}$ which fixes the points $0, \infty \in \mathbb{P}^{1}$ lifts to an additional $\mathbb{C}^{*}$-action on $N$ fixing $N_{\infty}$.

The equivariant cohomology $\operatorname{ring} H_{\mathbf{T}}^{*}(\bullet)$ is generated by the Chern classes $s_{1}, s_{2}$, and $s_{3}$ of the standard representation of the three $\mathbb{C}^{*}$ factors. At the $\mathbf{T}$-fixed point of $N$ over $0 \in \mathbb{P}^{1}$ the tangent weights are specified as follows

(i) tangent weights of $-s_{1}$ and $-s_{2}$ along the fiber directions for the action of $T$,

(ii) tangent weight $-s_{3}$ along $\mathbb{P}^{1}$ for the action on $\mathbb{C}^{*}$.

For the $\mathbf{T}$-fixed point of $N$ over $\infty \in \mathbb{P}^{1}$, the weights are $-s_{1},-s_{2}, s_{3}$. We define

(3) $\mathrm{Z}_{d, \eta}^{\mathrm{cap}}\left(\prod_{j=1}^{\ell} \tau_{i_{j}}\left(\gamma_{j}\right)\right)^{\mathbf{T}}=\sum_{n \in \mathbb{Z}} q^{n} \int_{\left[P_{n}\left(N / N_{\infty}, d\right)\right] \text { vir }} \prod_{j=1}^{\ell} \tau_{i_{j}}\left(\gamma_{j}\right) \cup \epsilon_{\infty}^{*}\left(\mathrm{C}_{\eta}\right)$,

by $\mathbf{T}$-equivariant residues where $\gamma_{j} \in H_{\mathbf{T}}^{*}\left(\mathbb{P}^{1}, \mathbb{Z}\right)$.

1.2. Reduction for the cap. Consider the following partition function for the cap

$$
\mathrm{Z}_{d, \eta}^{\mathrm{cap}}\left(\tau_{k}([0]) \cdot \prod_{j=1}^{\ell} \tau_{i_{j}}\left(\gamma_{j}\right)\right)^{\mathbf{T}}
$$

where $\gamma_{j} \in H_{\mathbf{T}}^{*}\left(\mathbb{P}^{1}, \mathbb{Z}\right)$. 
DESCENDENTS ON LOCAL CURVES: $\quad$ STATIONARY THEORY 7

The T-equivariant localization formula for (4) has two sides. The contribution over $0 \in \mathbb{P}^{1}$ yields the descendent vertex $\mathrm{W}_{\mu}^{\text {Vert }}$ of Section 2.6 of 20 . We will follow here exactly the terminology of the $\mathbf{T}$-fixed point analysis of Sections 2.1-2.7 of [20]. The contribution over $\infty \in \mathbb{P}^{1}$ yields rubber integrals discussed in Section 3.3 of [20]. While only the descendent vertex is required for the proof of Theorem 1, the rubber theory plays an essential role in the proof of Theorem 3.

Let $Q_{U}$ determine a $\mathbf{T}$-fixed point of the moduli space of stable pairs on the affine chart associated to $0 \in \mathbb{P}^{1}$. For each $x_{1}^{a} x_{2}^{b} \in \mu\left[x_{1}, x_{2}\right]$, let $c_{a, b}$ be the largest integer satisfying

$$
x_{1}^{a} x_{2}^{b} x_{3}^{-c_{a, b}} \in Q_{U} .
$$

The length of $Q_{U}$ is the sum of the $c_{a, b}$,

$$
\ell\left(Q_{U}\right)=\sum_{(a, b) \in \mu} c_{a, b} .
$$

The Laurent polynomial

$$
\mathrm{F}_{U}=\frac{1}{1-t_{3}} \sum_{(a, b) \in \mu} t_{1}^{a} t_{2}^{b} t_{3}^{-c_{a, b}}
$$

plays a basic role.

In the formula in Section 2.6 of [20] for the descendent vertex $\mathbf{W}_{\mu}^{\text {Vert }}\left(\tau_{k}([0])\right)$, the descendent2 $\tau_{k}([0])$ enters via

$$
\begin{aligned}
& \frac{1}{s_{1} s_{2}} \operatorname{ch}_{2+k}\left(\mathrm{~F}_{U} \cdot\left(1-t_{1}\right)\left(1-t_{2}\right)\left(1-t_{3}\right)\right)= \\
& \frac{1}{s_{1} s_{2}} \operatorname{ch}_{2+k}\left(\left(1-t_{1}\right)\left(1-t_{2}\right) \sum_{(a, b) \in \mu} t_{1}^{a} t_{2}^{b} t_{3}^{-c_{a, b}}\right)= \\
& \quad \frac{1}{s_{1} s_{2}} \operatorname{Coeff}_{z^{2+k}}\left(\left(1-e^{z s_{1}}\right)\left(1-e^{z s_{2}}\right) \sum_{(a, b) \in \mu} e^{z\left(a s_{1}+b s_{2}-c_{a, b} s_{3}\right)}\right) .
\end{aligned}
$$

The third line exhibits the action of the descendent on $Q_{U}$ as a symmetric function of the $d=|\mu|$ variables

$$
\left\{a s_{1}+b s_{2}-c_{a, b} s_{3} \mid(a, b) \in \mu\right\}
$$

with coefficients in $\mathbb{Q}\left[s_{1}, s_{2}\right]$.

In fact, the descendent $\tau_{k}([0])$ is a symmetric function of degree $k$ in the variables (6). The symmetric function is inhomogeneous with degree $k$ part equal to $\frac{\mathfrak{p}_{k}}{k !}$ where $\mathfrak{p}_{k}$ is the power sum. Since the ring of

\footnotetext{
${ }^{2}$ Here, the class $[0]$ is the pull-back to $N$ of the fixed point $0 \in \mathbb{P}^{1}$.
} 
symmetric functions in $d$ variables is generated by $\mathfrak{p}_{1}, \ldots, \mathfrak{p}_{d}$, we obtain universal reduction rules.

Let $\mathfrak{t}_{k}$ be the symmetric function in $d$ variables with coefficients in $\mathbb{Q}\left[s_{1}, s_{2}\right]$ defined by

$$
\sum_{k=0}^{\infty} \mathfrak{t}_{k} z^{k+2}=\frac{1}{s_{1} s_{2}}\left(1-e^{z s_{1}}\right)\left(1-e^{z s_{2}}\right) \sum_{n=0}^{\infty} \mathfrak{p}_{n} \frac{z^{n}}{n !} .
$$

For $k>d$, there are unique polynomials $f_{k, d}$ with coefficients in $\mathbb{Q}\left(s_{1}, s_{2}\right)$ satisfying

$$
\mathfrak{t}_{k}=f_{k, d}\left(\mathfrak{t}_{1}, \ldots, \mathfrak{t}_{d}\right) .
$$

We have proven the following result.

Proposition 1. In the degree $d$ theory of the $\mathbf{T}$-equivariant cap, the reduction rule

$$
\tau_{k}([0]) \mapsto f_{k, d}\left(\tau_{1}([0]), \ldots, \tau_{d}([0])\right)
$$

holds universally when $k>d$.

Explicitly, Proposition 1 1 yields the following equality for $\mathbf{T}$-equivariant integrals:

$$
\mathrm{Z}_{d, \eta}^{\text {cap }}\left(\tau_{k}([0]) \cdot \prod_{j=1}^{\ell} \tau_{i_{j}}\left(\gamma_{j}\right)\right)^{\mathbf{T}}=\mathrm{Z}_{d, \eta}^{\mathrm{cap}}\left(f_{k, d}\left(\tau_{1}(\mathrm{p}), \ldots, \tau_{d}(\mathrm{p})\right) \cdot \prod_{j=1}^{\ell} \tau_{i_{j}}\left(\gamma_{j}\right)\right)^{\mathbf{T}}
$$

for $k>d$. Of course, Proposition 1 implies the same result for the $T$-equivariant theory of the cap.

1.3. Proof of Theorem 1. Consider the partition function for the relative geometry $N / S$ over a curve $C$,

$$
\mathrm{Z}_{d, \eta^{1}, \ldots, \eta^{r}}^{N / S}\left(\tau_{k}(\mathrm{p}) \cdot \prod_{j=1}^{\ell} \tau_{i_{j}}\left(\gamma_{j}\right)\right)^{T}, \quad \gamma_{j} \in H^{*}(C, \mathbb{Z})
$$

Since the insertion $\tau_{k}(\mathrm{p})$ may be degenerated to lie on a cap, Proposition 1 implies Theorem 1 .

1.4. Parity considerations. We will need the following property of the reduction polynomials $f_{k, d}$ to obtain the functional equation of Theorem 2 ,

Lemma 1. For every $k>d>0$, the reduction polynomial

$$
f_{k, d} \in \mathbb{Q}\left(s_{1}, s_{2}\right)\left[x_{1}, \ldots, x_{d}\right]
$$


DESCENDENTS ON LOCAL CURVES: STATIONARY THEORY 9

lies in the span of the monomials of the form $x_{1}^{\sigma_{1}} \cdots x_{d}^{\sigma_{d}}$ where

$$
\sum_{i=1}^{d} i \sigma_{i} \equiv k \quad \bmod 2
$$

Proof. Using the homogeneity of $\mathfrak{t}_{i}$, we see from (7) the coefficient of $x_{1}^{\sigma_{1}} \cdots x_{d}^{\sigma_{d}}$ in $f_{k, d}$ is homogeneous as a rational function in $s_{1}$ and $s_{2}$. Moreover the degree of the coefficient is congruent $\bmod 2$ to $k-\sum_{i=1}^{d} i \sigma_{i}$. We need only show that these degrees are all even.

We write the descendent $\tau_{k}([0])$ as a symmetric function in the adjusted variables

$$
\left\{a s_{1}+b s_{2}-c_{a, b} s_{3}+\frac{s_{1}+s_{2}}{2} \mid(a, b) \in \mu\right\} .
$$

If we let $\mathfrak{p}_{k}^{\prime}$ denote the $k$ th power sum of these $d$ variables, then we have

$$
\sum_{k=0}^{\infty} \mathfrak{t}_{k} z^{k+2}=\frac{1}{s_{1} s_{2}}\left(e^{z s_{1} / 2}-e^{-z s_{1} / 2}\right)\left(e^{z s_{2} / 2}-e^{-z s_{2} / 2}\right) \sum_{n=0}^{\infty} \mathfrak{p}_{n}^{\prime} \frac{z^{n}}{n !},
$$

where $\mathfrak{t}_{k}$ is as in the proof of Proposition 1. Since

$$
\left(e^{z s_{1} / 2}-e^{-z s_{1} / 2}\right)\left(e^{z s_{2} / 2}-e^{-z s_{2} / 2}\right)
$$

is an even function of $s_{1}$ and $s_{2}$, the coefficients of the monomial of $f_{k, d}$ must have even degree.

\section{FACTORIZATION AND RATiOnALity}

2.1. Dependence upon the cap. Consider the stationary series

$$
\mathrm{Z}_{d, \eta^{1}, \ldots, \eta^{r}}^{N / S}\left(\prod_{j=1}^{\ell} \tau_{i_{j}}(\mathrm{p})\right)^{T}
$$

If $\ell=0$, then no descendents appear and the rationality of the partition function (8) has been proven in [15, 18]. If $\ell>0$, each stationary descendent $\tau_{i}(\mathrm{p})$ can be degenerated to a distinct cap. Hence, the series (8) is determined by:

- the stable pairs theory of local curves (without insertions),

- the 1-pointed caps $Z_{d, \eta}^{\text {cap }}\left(\tau_{k}(\mathrm{p})\right)^{T}$.

In fact, we can do much better by using Theorem 1, 
2.2. Factorization I. If $k>d$, then we have

$$
\mathrm{Z}_{d, \eta}^{\mathrm{cap}}\left(\tau_{k}(\mathrm{p})\right)^{T}=\mathrm{Z}_{d, \eta}^{\mathrm{cap}}\left(f_{k, d}\left(\tau_{1}(\mathrm{p}), \ldots, \tau_{d}(\mathrm{p})\right)\right)^{T}
$$

by Theorem 1. After expanding $f_{k, d}\left(\tau_{1}(\mathrm{p}), \ldots, \tau_{d}(\mathrm{p})\right)$ and degenerating each stationary descendent $\tau_{i}(\mathrm{p})$ to a distinct cap, we find the series (9) is determined by:

- the stable pairs theory of local curves (without insertions),

- the 1-pointed caps $\mathbf{Z}_{d, \eta}^{\mathrm{cap}}\left(\tau_{k \leq d}(\mathbf{p})\right)^{T}$.

2.3. Factorization II. We can further restrict the descendents $\tau_{k}(p)$ which occur on the caps by geometrically factoring the parts of the relative condition $\eta$.

Proposition 2. The series $\mathbf{Z}_{d, \eta}^{\mathrm{cap}}\left(\tau_{k \leq d}(\mathbf{p})\right)^{T}$ are determined by

- the stable pairs theory of local curves (without insertions),

- the 1-pointed caps $\mathbf{Z}_{c,(c)}^{\mathrm{cap}}\left(\tau_{c}(\mathrm{p})\right)^{T}$ for $1 \leq c \leq d$.

Proof. We proceed by induction on $d$. If $d=1$, there is nothing to prove. Assume Proposition 2 holds for all degrees less than $d$ and consider

$$
\mathbf{Z}_{d, \eta}^{\text {cap }}\left(\tau_{k}(\mathrm{p})\right)^{T}
$$

There are two main cases.

Case $k<d$.

We consider the geometry of $\mathbb{P}^{2} \times \mathbb{P}^{1}$ relative to the fiber

$$
\mathbb{P}_{\infty}^{2}=\mathbb{P}^{2} \times\{\infty\} \subset \mathbb{P}^{2} \times \mathbb{P}^{1}
$$

Let $\beta \in H_{2}\left(\mathbb{P}^{2} \times \mathbb{P}^{1}, \mathbb{Z}\right)$ be the class of the section $\mathbb{P}^{1}$ contracted over $\mathbb{P}^{2}$. The 2-dimensional torus $T$ acts on $\mathbb{P}^{2}$ with fixed points $\xi_{0}, \xi_{1}, \xi_{2} \in \mathbb{P}^{2}$. The tangent weights can be chosen as follows:

$$
-s_{1},-s_{2} \text { for } \xi_{0}, s_{1}, s_{1}-s_{2} \text { for } \xi_{1}, s_{2}-s_{1}, s_{2} \text { for } \xi_{2} .
$$

Let the partition $\eta$ have parts $\eta_{1}, \ldots, \eta_{\ell}$. Let $\widetilde{\eta}$ be the cohomology weighted partition with $\eta_{1}$ of weight $\left[\xi_{0}\right] \in H^{*}\left(\mathbb{P}^{2}, \mathbb{Z}\right)$ and all of the other parts assigned weight $1 \in H_{T}^{*}\left(\mathbb{P}^{2}, \mathbb{Z}\right)$. The series

$$
\mathbf{Z}_{d \beta, \widetilde{\eta}}^{\mathbb{P}^{2} \times \mathbb{P}^{1} / \mathbb{P}_{\infty}^{2}}\left(\tau_{k}([0])\right)^{T} \in \mathbb{Q}\left[s_{1}, s_{2}\right][[q]]
$$

is well-defined. 
DESCENDENTS ON LOCAL CURVES: STATIONARY THEORY 11

The virtual dimension of the moduli space $P_{n}\left(\mathbb{P}^{2} \times \mathbb{P}^{1} / \mathbb{P}_{\infty}^{2}, d \beta\right)$ after the imposition of the boundary condition $\tilde{\eta}$ is

$$
2 d-2-\sum_{i=1}^{\ell}\left(\eta_{i}-1\right)=d+\ell-2 \geq d-1 .
$$

The dimension of the integrand $\tau_{k}([0])$ is $k<d$. Hence, the integrals

$$
\left\langle\tau_{k}([0])\right)_{n, d \beta}^{\mathbb{P}^{2} \times \mathbb{P}^{1}, \widetilde{\eta}}=\int_{\left[P_{n}\left(\mathbb{P}^{2} \times \mathbb{P}^{1} / \mathbb{P}_{\infty}^{2}, d \beta\right)\right]} \tau_{k}([0]) \cup \epsilon^{*}\left(\mathrm{C}_{\tilde{\eta}}\right)
$$

arising as coefficients of (10) have degree at most 0 in $\mathbb{Q}\left[s_{1}, s_{2}\right]$. If the degree is negative, then the series (10) vanishes.

The degree of (10) is 0 only when $k=d-1$ and $\eta=(d)$. The moduli space then lies entirely in

$$
\mathbb{C}^{2} \times \mathbb{P}^{1} \subset \mathbb{P}^{2} \times \mathbb{P}^{1}
$$

where $\mathbb{C}^{2} \subset \mathbb{P}^{2}$ is the $T$-invariant affine containing $\xi_{0}$ (corresponding to the cohomology weight $\left[\xi_{0}\right]$ on the part $\left.d\right)$. By the basic divisibility results of [15, 18], the linear factor $s_{1}+s_{2}$ must divide the $q^{n}$ coefficient of (10) for all $n>d$. Since the invariant is of degree 0 , the divisibility by $s_{1}+s_{2}$ is impossible unless all such coefficients vanish. Since the leading term of (10) is $q^{d}$, we conclude (10) is a monomial in $q$.

If $k<d$, we have calculated the series (10). Direct calculation of (10) by $T$-equivariant localization yields a single term equal to

$$
\mathrm{Z}_{d, \eta}^{\text {cap }}\left(\tau_{k}(\mathrm{p})\right)^{T}
$$

up to an $s_{1} s_{2}$ factor. The $T$-equivariant localization formula for the relative geometry $\mathbb{P}^{2} \times \mathbb{P}^{1} / \mathbb{P}_{\infty}^{2}$ in the class $d \beta$ distributes the parts of $\tilde{\eta}$ among the $T$-fixed points

$$
\xi_{0}, \xi_{1}, \xi_{2} \in \mathbb{P}^{2}
$$

The term equal to (11) arises when all parts are distributed to $\xi_{0}$. Since the first part of $\widetilde{\eta}$ must be distributed to $\xi_{0}$, the remaining terms are known by the induction hypothesis. Hence, we have calculated (11).

Case $\ell>1$.

The dimension estimates as above show the series

$$
\mathrm{Z}_{d \beta, \widetilde{\eta}}^{\mathbb{P}^{2} \times \mathbb{P}^{1} / \mathbb{P}_{\infty}^{2}}\left(\tau_{k}([0])\right)^{T} \in \mathbb{Q}\left[s_{1}, s_{2}\right][[q]]
$$

is degree at most 0 in $s_{1}$ and $s_{2}$. The series (12) must vanish in the negative degree case.

The degree of (12) is 0 only when $k=d$ and $\eta=\left(d_{1}, d_{2}\right)$. In the degree 0 case, the invariant (12) is independent of $s_{1}$ and $s_{2}$, so we may 
calculate (12) in the specialization $s_{1}+s_{2}=0$. In the $T$-equivariant localization of (12), the terms at $\xi_{0}$ all have vanishing coefficients of $q^{n>d}$ in the specialization $s_{1}+s_{2}=0$. The terms away from $\xi_{0}$ are known inductively. Hence, (12) is determined.

If $\ell>1$, we have calculated the series (12). As before, the $T$ equivariant localization formula for (12) yields a single term equal to $\mathrm{Z}_{d, \eta}^{\text {cap }}\left(\tau_{k}(\mathrm{p})\right)^{T}$ up to an $s_{1} s_{2}$ factor. The remaining terms are known by the induction hypothesis. We have calculated $Z_{d, \eta}^{\text {cap }}\left(\tau_{k}(\mathbf{p})\right)^{T}$.

The only possibility not covered by the two above cases is the 1pointed cap

$$
\mathbf{Z}_{d,(d)}^{\text {cap }}\left(\tau_{d}(\mathrm{p})\right)^{T} \in \mathbb{Q}\left[s_{1}, s_{2}\right][[q]] .
$$

The factorization methods do not inductively determine (13).

2.4. Proof of Theorem 2. The methods of Sections 2.2 2.3 provide an effective algorithm for calculating an arbitrary degree $d$ stationary series (8) in terms of

- the stable pairs theory of local curves (without insertions),

- the 1-pointed caps $\mathrm{Z}_{c,(c)}^{\mathrm{cap}}\left(\tau_{c}(\mathrm{p})\right)^{T}$ for $1 \leq c \leq d$.

The partition functions of the stable pairs theory of local curves (without insertions) are rational and satisfy the functional equation of Theorem [2, see Theorems 2 and 3 of [18]. The steps in the effective algorithm preserve the functional equation. For the Factorization I step, Lemma 1 is needed to ensure that the total weight of the descendent insertions does not change parity. Theorem 2 then follows from Theorem 3 proven in Section 4 below together with the observation that the rational functions appearing there satisfy the functional equation.

\section{LOCALIZATION FORMALISM}

3.1. Formula. The T-equivariant localization formula for the capped 1-leg descendent vertex is the following:

$$
\mathbf{Z}_{d, \eta}^{\text {cap }}\left(\prod_{i=1}^{k} \tau_{i_{j}}([0])\right)^{\mathbf{T}}=\sum_{|\mu|=d} \mathbf{W}_{\mu}^{\text {Vert }}\left(\prod_{j=1}^{k} \tau_{i_{j}}([0])\right) \cdot \mathbf{W}_{\mu}^{(0,0)} \cdot \mathbf{S}_{\eta}^{\mu} .
$$

The result is a consequence of [6] applied to stable pairs theory of the cap [23] - see Section 3.4 of [20]. The form is the same as the Donaldson-Thomas localization formulas used in [11, 18].

The right side of localization formula is expressed in term of three parts of different geometric origins: 
- the vertex term $\mathbf{W}_{\mu}^{\text {Vert }}\left(\prod_{j=1}^{k} \tau_{i_{j}}([0])\right)$ over $0 \in \mathbb{P}^{1}$,

- the edge term $\mathbf{W}_{\mu}^{(0,0)}$,

- the rubber integrals $\mathrm{S}_{\eta}^{\mu}$ over $\infty \in \mathbb{P}^{1}$.

The vertex term has been explained (for $i=1$ ) already in Section 1.2. The edge term $\mathrm{W}_{\mu}^{(0,0)}$ is simply the inverse product of the tangent weights of the Hilbert scheme of points of $\mathbb{C}^{2}$ at the $T$-fixed point corresponding to the partition $\mu$. We review the rubber integrals here.

3.2. Rubber theory. The stable pairs theory of rubben naturally arises at the boundary of $P_{n}\left(N / N_{\infty}, d\right)$. Let $R$ be a rank 2 bundle of level $(0,0)$ over $\mathbb{P}^{1}$. Let

$$
R_{0}, R_{\infty} \subset R
$$

denote the fibers over $0, \infty \in \mathbb{P}^{1}$. The 1 -dimensional torus $\mathbb{C}^{*}$ acts on $R$ via the symmetries of $\mathbb{P}^{1}$. Let $P_{n}\left(R / R_{0} \cup R_{\infty}, d\right)$ be the relative moduli space of ideal sheaves, and let

$$
P_{n}\left(R / R_{0} \cup R_{\infty}, d\right)^{\circ} \subset P_{n}\left(R / R_{0} \cup R_{\infty}, d\right)
$$

denote the open set with finite stabilizers for the $\mathbb{C}^{*}$-action and no destabilization over $\infty \in \mathbb{P}^{1}$. The rubber moduli space,

$$
P_{n}\left(R / R_{0} \cup R_{\infty}, d\right)^{\sim}=P_{n}\left(R / R_{0} \cup R_{\infty}, d\right)^{\circ} / \mathbb{C}^{*},
$$

denoted by a superscripted tilde, is determined by the (stack) quotient. The moduli space is empty unless $n>d$. The rubber theory of $R$ is defined by integration against the rubber virtual class,

$$
\left[P_{n}\left(R / R_{0} \cup R_{\infty}, d\right)^{\sim}\right]^{v i r} .
$$

All of the above rubber constructions are $T$-equivariant for the scaling action on the fibers of $R$ with weights $s_{1}$ and $s_{2}$.

The rubber moduli space $P_{n}\left(R / R_{0} \cup R_{\infty}, d\right)^{\sim}$ carries a cotangent line at the dynamical point $0 \in \mathbb{P}^{1}$. Let

$$
\psi_{0} \in A_{T}^{1}\left(P_{n}\left(R / R_{0} \cup R_{\infty}, d\right)^{\sim}, \mathbb{Q}\right)
$$

denote the associated cotangent line class. Let

$$
\mathrm{P}_{\mu} \in A_{T}^{2 d}\left(\operatorname{Hilb}\left(\mathbb{C}^{2}, d\right), \mathbb{Z}\right)
$$

be the class corresponding to the $T$-fixed point determined by the monomial ideal $\mu\left[x_{1}, x_{2}\right] \subset \mathbb{C}\left[x_{1}, x_{2}\right]$.

\footnotetext{
${ }^{3}$ We follow the terminology and conventions of the parallel rubber discussion for the local Donaldson-Thomas theory of curves treated in 18.
} 
In the localization formula for the cap, special rubber integrals with relative conditions $\mathrm{P}_{\mu}$ over 0 and $\mathrm{C}_{\eta}$ (in the Nakajima basis) over $\infty$ arise. Let

$$
\mathrm{S}_{\eta}^{\mu}=\sum_{n \geq d} q^{n}\left\langle\mathrm{P}_{\mu}\left|\frac{1}{s_{3}-\psi_{0}}\right| \mathrm{C}_{\eta}\right\rangle_{n, d}^{\sim} \in \mathbb{Q}\left(s_{1}, s_{2}, s_{3}\right)((q)) .
$$

The bracket on the right is the rubber integral defined by $T$-equivariant residues. If $n=d$, the rubber moduli space in undefined - the bracket is then taken to be the $T$-equivariant intersection pairing between the classes $\mathrm{P}_{\mu}$ and $\mathrm{C}_{\eta}$ in $\operatorname{Hilb}\left(\mathbb{C}^{2}, d\right)$.

\section{Calculation of $Z_{d,(d)}^{\text {cap }}\left(\tau_{d}(\mathrm{p})\right)^{\mathbf{T}}$}

4.1. Dimension. The notation $(d[0])$ will be used to assign the weight $[0] \in A_{T}^{*}\left(\mathbb{C}^{2}, \mathbb{Q}\right)$ to the part $d$. Since

$$
[0]=s_{1} s_{2} \in A_{T}^{*}\left(\mathbb{C}^{2}, \mathbb{Q}\right),
$$

we see 4

$$
\mathrm{Z}_{d,(d)}^{\text {cap }}\left(\tau_{d}(\mathrm{p})\right)^{\mathbf{T}}=\left(\frac{1}{s_{1} s_{2}}\right) \mathbf{Z}_{d,(d[0])}^{\text {cap }}\left(\tau_{d}(\mathbf{p})\right)^{\mathbf{T}}
$$

After imposing the boundary condition $(d[0])$, the moduli space

$$
P_{n}\left(\mathbb{P}^{2} \times \mathbb{P}^{1} / \mathbb{P}_{\infty}^{2}, d \beta\right)
$$

is compact of virtual dimension $d-1$.

The moduli space $P_{n}\left(\mathbb{P}^{2} \times \mathbb{P}^{1} / \mathbb{P}_{\infty}^{2}, d \beta\right)$ is empty for $n<d$ and isomorphic to $\operatorname{Hilb}\left(\mathbb{C}^{2}, d\right)$ for $n=d$. Hence, the leading term of the series $\mathbf{Z}_{d,(d[0])}^{\text {cap }}\left(\tau_{d}(\mathbf{p})\right)^{\mathbf{T}}$ is the classical pairing

$$
q^{d}\left\langle\tau_{d}, \mathrm{C}_{(d[0])}\right\rangle_{\operatorname{Hilb(\mathbb {C}^{2},d)}} \in \mathbb{Q}\left[s_{1}, s_{2}\right] .
$$

The class $\tau_{d}$ is defined as follows. Let $\mathbb{F}_{0}$ be the universal quotient sheaf on $\operatorname{Hilb}\left(\mathbb{C}^{2}, d\right) \times \mathbb{C}^{2}$. Then,

$$
\tau_{d}=\pi_{*}\left(\operatorname{ch}_{2+d}\left(\mathbb{F}_{0}\right)\right) \in A_{T}^{d}\left(\operatorname{Hilb}\left(\mathbb{C}^{2}, d\right)\right)
$$

where $\pi$ is the projection

$$
\pi: \operatorname{Hilb}\left(\mathbb{C}^{2}, d\right) \times \mathbb{C}^{2} \rightarrow \operatorname{Hilb}\left(\mathbb{C}^{2}, d\right) .
$$

Lemma 2. $\mathbf{Z}_{d,(d[0])}^{\text {cap }}\left(\tau_{d}(\mathrm{p})\right)^{\mathbf{T}}=\left(s_{1}+s_{2}\right) \cdot q^{d} F(d)(q)$ for $F(d) \in \mathbb{Q}[[q]]$.

\footnotetext{
${ }^{4}$ We will consider descendents here equivariant with respect to the 3 -torus $\mathbf{T}$ of Section 1
} 
DESCENDENTS ON LOCAL CURVES: STATIONARY THEORY 15

Proof. By compactness of the underlying moduli spaces of pairs, we see the series $\mathbf{Z}_{d,(d[0])}^{\text {cap }}\left(\tau_{d}(\mathbf{p})\right)^{\mathbf{T}}$ must lie in $\mathbb{Q}\left[s_{1}, s_{2}, s_{3}\right][[q]]$. The leading $q^{d}$ coefficient certainly has no $s_{3}$ dependence by (15). By dimension considerations, the leading $q^{d}$ coefficient must be linear and thus, by symmetry, a multiple of $s_{1}+s_{2}$. For the coefficient of $q^{n>d}$, divisibility by $s_{1}+s_{2}$ is obtained from [15, 18].

4.2. Localization. We wish to compute the series

$$
F(d)=\frac{s_{1} s_{2}}{s_{1}+s_{2}} q^{-d} \mathrm{Z}_{d,(d)}^{\mathrm{cap}}\left(\tau_{d}([0])\right)^{\mathbf{T}} \in \mathbb{Q}[[q]] \subset \mathbb{Q}\left(s_{1}, s_{2}, s_{3}\right)[[q]]
$$

introduced in Lemma 2, Via the localization formula (14), we have

$$
F(d)=\frac{s_{1} s_{2}}{s_{1}+s_{2}} q^{-d} \sum_{|\mu|=d} \mathrm{~W}_{\mu}^{\operatorname{Vert}}\left(\tau_{d}([0])\right) \cdot \mathrm{W}_{\mu}^{(0,0)} \cdot \mathrm{S}_{(d)}^{\mu} .
$$

We will separate the classical terms occuring on the right side.

By definition, the classical term of $\mathbf{W}_{\mu}^{\text {Vert }}\left(\tau_{d}([0])\right.$ is the leading $q^{d}$ term. Let

$$
\mathrm{F}_{\mu}=\sum_{(a, b) \in \mu} t_{1}^{a} t_{2}^{b}
$$

We write the vertex as

$$
\mathrm{W}_{\mu}^{\text {Vert }}\left(\tau_{d}([0])\right)=\frac{q^{d}}{s_{1} s_{2}} \operatorname{ch}_{d+2}\left(\mathrm{~F}_{\mu} \cdot\left(1-t_{1}\right)\left(1-t_{2}\right)\right)+\widehat{\mathbf{W}}_{\mu}^{\text {Vert }}\left(\tau_{d}([0])\right)
$$

where $\widehat{\mathbf{W}}_{\mu}^{\text {Vert }}\left(\tau_{d}([0])\right)$ represents all the higher order terms in $q$. Similarly, we write

$$
\mathrm{S}_{(d)}^{\mu}=\left\langle\mathrm{P}_{\mu}, \mathrm{C}_{(d)}\right\rangle+\widehat{\mathrm{S}}_{(d)}^{\mu}
$$

where the leading term $\left\langle\mathrm{P}_{\mu}, \mathrm{C}_{(d)}\right\rangle$ is the $T$-equivariant pairing on $\operatorname{Hilb}\left(\mathbb{C}^{2}, d\right)$.

Using the above formulas with the leading classical terms, we rewrite the result of the localization formula as

$$
\begin{aligned}
F(d)= & \sum_{|\mu|=d} \frac{1}{s_{1}+s_{2}} \operatorname{ch}_{d+2}\left(\mathrm{~F}_{\mu} \cdot\left(1-t_{1}\right)\left(1-t_{2}\right)\right) \cdot \mathrm{W}_{\mu}^{(0,0)} \cdot\left\langle\mathrm{P}_{\mu}, \mathrm{C}_{(d)}\right\rangle \\
& +\sum_{|\mu|=d} \frac{s_{1} s_{2}}{s_{1}+s_{2}} q^{-d} \widehat{\mathrm{W}}_{\mu}^{\mathrm{Vert}}\left(\tau_{d}([0])\right) \cdot \mathrm{W}_{\mu}^{(0,0)} \cdot\left\langle\mathrm{P}_{\mu}, \mathrm{C}_{(d)}\right\rangle \\
& +\sum_{|\mu|=d} \frac{1}{s_{1}+s_{2}} \operatorname{ch}_{d+2}\left(\mathrm{~F}_{\mu} \cdot\left(1-t_{1}\right)\left(1-t_{2}\right)\right) \cdot \mathrm{W}_{\mu}^{(0,0)} \cdot \widehat{\mathrm{S}}_{(d)}^{\mu} \\
& +\sum_{|\mu|=d} \frac{s_{1} s_{2}}{s_{1}+s_{2}} q^{-d} \widehat{\mathrm{W}}_{\mu}^{\mathrm{Vert}}\left(\tau_{d}([0])\right) \cdot \mathrm{W}_{\mu}^{(0,0)} \cdot \widehat{\mathrm{S}}_{(d)}^{\mu} \cdot
\end{aligned}
$$


The first line on the right is the classical pairing

$$
F_{0}(d)=\frac{1}{s_{1}+s_{2}}\left\langle\tau_{d}, \mathrm{C}_{(d[0])}\right\rangle \in \mathbb{Q}
$$

which we will compute in Proposition 3 below. We will compute the difference

$$
\widehat{F}(d)=F(d)-F_{0}(d)
$$

by evaluating each of the other three terms at $s_{2}=-s_{1}$, expanding as a Laurent series in $\frac{s_{3}}{s_{1}}$, and taking the constant term.

Both $\widehat{\mathrm{W}}_{\mu}^{\text {Vert }}$ and $\widehat{\mathrm{S}}_{(d)}^{\mu}$ are divisible by $s_{1}+s_{2}$. Therefore, the fourth term in the formula for $F(d)$ vanishes after the substitution $s_{2}=-s_{1}$. Only two terms,

$$
\begin{aligned}
& \widehat{F}(d)=\left.\sum_{|\mu|=d}\left(\frac{s_{1} s_{2}}{s_{1}+s_{2}} q^{-d} \widehat{\mathrm{W}}_{\mu}^{\mathrm{Vert}}\left(\tau_{d}([0])\right) \cdot \mathrm{W}_{\mu}^{(0,0)} \cdot\left\langle\mathrm{P}_{\mu}, \mathrm{C}_{(d)}\right\rangle\right)\right|_{s_{2}=-s_{1}} \\
& \quad+\left.\sum_{|\mu|=d}\left(\frac{1}{s_{1}+s_{2}} \mathrm{ch}_{d+2}\left(\mathrm{~F}_{\mu} \cdot\left(1-t_{1}\right)\left(1-t_{2}\right)\right) \cdot \mathrm{W}_{\mu}^{(0,0)} \cdot \widehat{\mathrm{S}}_{(d)}^{\mu}\right)\right|_{s_{2}=-s_{1}}
\end{aligned}
$$

remain.

We evaluate the two above terms separately. The first requires detailed knowledge of the vertex factor

$$
\left.\frac{s_{1} s_{2}}{s_{1}+s_{2}} q^{-d} \widehat{\mathrm{W}}_{\mu}^{\mathrm{Vert}}\left(\tau_{d}([0])\right)\right|_{s_{2}=-s_{1}}
$$

and is evaluated in Section 4.3. The second requires detailed knowledge of the rubber factor

$$
\left.\frac{1}{s_{1}+s_{2}} \widehat{\mathrm{S}}_{(d)}^{\mu}\right|_{s_{2}=-s_{1}}
$$

and is evaluated in Section 4.4

4.3. Vertex calculation. We begin with the first term

$$
\left.\sum_{|\mu|=d}\left(\frac{s_{1} s_{2}}{s_{1}+s_{2}} q^{-d} \widehat{\mathrm{W}}_{\mu}^{\mathrm{Vert}}\left(\tau_{d}([0])\right) \cdot \mathrm{W}_{\mu}^{(0,0)} \cdot\left\langle\mathrm{P}_{\mu}, \mathrm{C}_{(d)}\right\rangle\right)\right|_{s_{2}=-s_{1}}
$$

of $\widehat{F}(d)$. The pairing $\left\langle\mathrm{P}_{\mu}, \mathrm{C}_{(d)}\right\rangle$ has a simple expression mod $s_{1}+s_{2}$,

$$
\left.\left\langle\mathrm{P}_{\mu}, \mathrm{C}_{(d)}\right\rangle\right|_{s_{2}=-s_{1}}=\frac{(-1)^{d-1}(d-1) !}{\operatorname{dim} \mu} \chi^{\mu}((d)) s_{1}^{d-1} .
$$

Here, $\operatorname{dim} \mu$ is the dimension of the irreducible representation of the symmetric group $\Sigma_{d}$ corresponding to the partition $\mu$, and $\chi^{\mu}$ is the associated character. The proof of (18) is obtained directly from the 
DESCENDENTS ON LOCAL CURVES: STATIONARY THEORY 17

Jack polynomial expression for the $T$-fixed points of $\operatorname{Hilb}\left(\mathbb{C}^{2}, d\right)$, see Section 3.7 of [17] 5

The character $\chi^{\mu}$ vanishes on a $d$-cycle unless $\mu$ is of the following simple form

$$
\alpha_{a}=(a+1,1, \ldots, 1)
$$

for $0 \leq a \leq d-1$. We have

$$
\chi^{\alpha_{a}}((d))=(-1)^{d-1-a} .
$$

We will restrict to the case $\mu=\alpha_{a}$ and replace the sum over $\mu$ with a sum over $a$. The dimension formula

$$
\operatorname{dim} \alpha_{a}=\left(\begin{array}{c}
d-1 \\
a
\end{array}\right)
$$

holds. The constant

$$
b=d-1-a
$$

will occur often below.

The edge factor $\mathbf{W}_{\mu}^{(0,0)}$ is also easy to compute after the evaluation $s_{2}=-s_{1}$ :

$$
\left.\mathbf{W}_{\mu}^{(0,0)}\right|_{s_{2}=-s_{1}}=\frac{(-1)^{d}(\operatorname{dim} \mu)^{2}}{(d !)^{2}} s_{1}^{-2 d} .
$$

The dimension of $\mu$ here enters via the hook length formula.

The most complicated part of the calculation is the vertex factor $\widehat{\mathrm{W}}_{\mu}^{\mathrm{Vert}}\left(\tau_{d}([0])\right)$ for $\mu=\alpha_{a}$. From Section 2.6 of [20],

$$
\begin{aligned}
\widehat{\mathrm{W}}_{\mu}^{\text {Vert }} & \left(\tau_{d}([0])\right)= \\
& \sum_{Q_{U}:} \frac{q^{d+l\left(Q_{U}\right)>0}}{s_{1} s_{2}} \operatorname{ch}_{d+2}\left(\mathrm{~F}_{U} \cdot\left(1-t_{1}\right)\left(1-t_{2}\right)\left(1-t_{3}\right)\right) \cdot e\left(-\mathrm{V}_{U}\right),
\end{aligned}
$$

where the sum runs over T-fixed loci $Q_{U}$ of positive length. Recall, the the $\mathbf{T}$-fixed loci correspond to box configurations defined by height functions $c_{a, b}$ on the partition $\mu$ determining $\mathrm{F}_{U}$ by formula (5). The term $\mathrm{V}_{U}$ is expressed in terms of $\mathrm{F}_{U}$ in Section 2.5 of [20].

In the case $\mu=\alpha_{a}$, a straightforward calculation shows the vertex weight $e\left(-\mathrm{V}_{U}\right)$ is divisible by $\left(s_{1}+s_{2}\right)^{2}$ unless the box configuration is a cylinder (of height $h>0$ ) under a rim hook $\eta$ of $\mu .6$ We break the

\footnotetext{
${ }^{5}$ Our variable conventions here differ slightly from [17]. Specifically, our $s_{i}$ correspond to $-t_{i}$ in 17 .

${ }^{6}$ The divisibility statement is actually true for any $\mu$.
} 
sum into terms by the size $r$ of $\eta$. When $r=d$, the only possibility for the rim hook is $\eta=\mu$. The corresponding vertex weight is

$$
\left.\frac{1}{s_{1}+s_{2}} e\left(-\mathrm{V}_{U}\right)\right|_{s_{2}=-s_{1}}=\frac{(-1)^{d h+1}}{h s_{3}}\left(1+\frac{h s_{3}}{s_{1}} \sum_{\substack{i=-b \\ i \neq 0}}^{a} \frac{1}{i}+\ldots\right) \text {. }
$$

Here and below, the dots on the right stand for terms of order 2 and higher in $\frac{s_{3}}{s_{1}}$. For each $r<d$, there are at most two such rim hooks, depending on whether $a \geq r$ and whether $d-1-a \geq r$. For $a \geq r$, we find

$$
\begin{aligned}
\left.\frac{1}{s_{1}+s_{2}} e\left(-\mathrm{V}_{U}\right)\right|_{s_{2}=-s_{1}} & = \\
& \quad \frac{(-1)^{r h+1}}{h s_{3}}\left(1+\frac{h s_{3}}{s_{1}}\left(\frac{1}{d}-\frac{1}{d-r}-\frac{1}{r}+\sum_{i=a-r+1}^{a} \frac{1}{i}\right)+\ldots\right) .
\end{aligned}
$$

For $d-1-a \geq r$, the answer is obtained by symmetry by interchanging $s_{1}$ and $s_{2}$. The symmetry propagates through the entire calculation of (17). After setting $s_{2}=-s_{1}$, we will take the constant term of the Laurent expansion in

$$
\frac{s_{3}}{s_{1}}=-\frac{s_{3}}{s_{2}} .
$$

Hence, we can treat the symmetry as exact.

After putting all the terms together and inserting the descendent factors, we obtain for

$$
\left.\frac{s_{1} s_{2}}{s_{1}+s_{2}} q^{-d} \mathbf{W}_{\mu}^{\operatorname{Vert}+}\left(\tau_{d}([0])\right)\right|_{s_{2}=-s_{1}}
$$

the following formula:

$$
\begin{gathered}
\sum_{h=1}^{\infty} \frac{(-1)^{d h+1} q^{d h}}{h s_{3}}\left(1+\frac{h s_{3}}{s_{1}} \sum_{\substack{i=-b \\
i \neq 0}}^{a} \frac{1}{i}+\ldots\right) \\
\cdot \operatorname{ch}_{d+2}\left(-t_{1}^{a+1} t_{3}^{-h}+t_{1}^{a} t_{3}^{-h}+t_{1}^{-b} t_{3}^{-h}-t_{1}^{-b-1} t_{3}^{-h}\right) \\
+2 \sum_{r=1}^{a} \sum_{h=1}^{\infty} \frac{(-1)^{r h+1} q^{r h}}{h s_{3}}\left(1+\frac{h s_{3}}{s_{1}}\left(\frac{1}{d}-\frac{1}{d-r}-\frac{1}{r}+\sum_{i=a-r+1}^{a} \frac{1}{i}\right)+\ldots\right) \\
\cdot \operatorname{ch}_{d+2}\left(-t_{1}^{a+1} t_{3}^{-h}+t_{1}^{a} t_{3}^{-h}+t_{1}^{a-r+1} t_{3}^{-h}-t_{1}^{a-r} t_{3}^{-h}\right)
\end{gathered}
$$

Here, we have included the symmetry discussed above.

After combining all of the parts of (17), summing over $\mu=\alpha_{a}$, and taking the constant term when expanded as a Laurent series in $\frac{s_{3}}{s_{1}}$, we 
obtain an expression of the form

$$
\sum_{r=1}^{d} A_{r} \frac{(-q)^{r}}{1-(-q)^{r}}
$$

The explicit formulas for $A_{r}$ depend upon two cases. For $A_{d}$, we have

$$
\begin{gathered}
(-1)^{d-1} d \cdot d !(d+2) ! A_{d}= \\
\sum_{a+b=d-1}(-1)^{a}\left(\begin{array}{c}
d-1 \\
a
\end{array}\right) \cdot \sum_{\substack{i=-b \\
i \neq 0}}^{a} \frac{1}{i}\left(-(-b-1)^{d+2}+(-b)^{d+2}+a^{d+2}-(a+1)^{d+2}\right) \\
-(d+2) \sum_{a+b=d-1}(-1)^{a}\left(\begin{array}{c}
d-1 \\
a
\end{array}\right)\left(-(-b-1)^{d+1}+(-b)^{d+1}+a^{d+1}-(a+1)^{d+1}\right),
\end{gathered}
$$

and for $r<d$, we have

$$
\begin{gathered}
\frac{(-1)^{d-1}}{2} d \cdot d !(d+2) ! A_{r}= \\
\sum_{\substack{a+b=d-1 \\
a \geq r}}(-1)^{a}\left(\begin{array}{c}
d-1 \\
a
\end{array}\right)\left(\frac{1}{d}-\frac{1}{d-r}-\frac{1}{r}+\sum_{i=a-r+1}^{a} \frac{1}{i}\right) \\
\cdot\left(-(-b-1)^{d+2}+(-b)^{d+2}+a^{d+2}-(a+1)^{d+2}\right) \\
-(d+2) \sum_{a=r}^{d-1}(-1)^{a}\left(\begin{array}{c}
d-1 \\
a
\end{array}\right)\left(-(a-r)^{d+1}+(a-r+1)^{d+1}+a^{d+1}-(a+1)^{d+1}\right) .
\end{gathered}
$$

While the above formulas for $A_{d}$ and $A_{r<d}$ look unpleasantly complicated, a remarkable cancellation will occur in Section 4.6.

4.4. Rubber calculation. Evaluating the second term

$$
\left.\sum_{|\mu|=d}\left(\frac{1}{s_{1}+s_{2}} \operatorname{ch}_{d+2}\left(\mathrm{~F}_{\mu} \cdot\left(1-t_{1}\right)\left(1-t_{2}\right)\right) \cdot \mathrm{W}_{\mu}^{(0,0)} \cdot \widehat{\mathrm{S}}_{(d)}^{\mu}\right)\right|_{s_{2}=-s_{1}}
$$

requires care in moving between two bases for the equivariant cohomology of the Hilbert scheme of $\mathbb{C}^{2}$ (which we identify with Fock space): the Nakajima basis $\left\{C_{\lambda}\right\}$ and the $T$-fixed point basis $\left\{P_{\lambda}\right\}$. The change of basis formula is simple $\bmod s_{1}+s_{2}$ :

$$
\mathrm{P}_{\lambda}=\sum_{\mu} \frac{(-1)^{\ell(\mu)} d !}{\operatorname{dim} \lambda} \chi^{\lambda}(\mu) s_{1}^{d+\ell(\mu)} \mathrm{C}_{\mu}
$$

Our $\widehat{\mathrm{S}}_{(d)}^{\mu}$ should be viewed as having upper index given in the $T$-fixed point basis but lower index in the Nakajima basis. 
The main tool for evaluating $S$ is the quantum differential equation of [18] valid also for stable pairs [15],

$$
s_{3} q \frac{d}{d q} \mathrm{~S}=\mathrm{MS}-\mathrm{SM}(0) \text {. }
$$

Here, $\mathbf{S}$ has both components indexed by the Nakajima basis and is viewed as an operator on Fock space, see [18]. The operator $\mathrm{M}$ is defined on Fock space by

$$
\begin{aligned}
\mathrm{M}\left(q, s_{1}, s_{2}\right)=\left(s_{1}+s_{2}\right) & \sum_{k>0} \frac{k}{2} \frac{(-q)^{k}+1}{(-q)^{k}-1} \alpha_{-k} \alpha_{k}+ \\
& \frac{1}{2} \sum_{k, l>0}\left[s_{1} s_{2} \alpha_{k+l} \alpha_{-k} \alpha_{-l}-\alpha_{-k-l} \alpha_{k} \alpha_{l}\right] .
\end{aligned}
$$

The $q$-dependence of $M$ is only in the first sum in (23). The operator $\mathrm{M}(0)$ is the $q^{0}$-coefficient of $\mathrm{M}$.

From the differential equation (22), we find

$$
s_{3} q \frac{d}{d q} \mathrm{~S}=\left(s_{1}+s_{2}\right) \mathrm{A}+[\mathrm{B}, \mathrm{S}] \quad \bmod \left(s_{1}+s_{2}\right)^{2} .
$$

The first term is

$$
\mathrm{A}=\left(\sum_{k>0} \frac{k}{2} \frac{(-q)^{k}+1}{(-q)^{k}-1} \alpha_{-k} \alpha_{k}\right) \circ \mathrm{S}(0)+\mathrm{S}(0) \circ\left(\sum_{k>0} \frac{k}{2} \alpha_{-k} \alpha_{k}\right) .
$$

The operator in the second term is

$$
\mathrm{B}=\frac{1}{2} \sum_{k, l>0}\left[s_{1} s_{2} \alpha_{k+l} \alpha_{-k} \alpha_{-l}-\alpha_{-k-l} \alpha_{k} \alpha_{l}\right] .
$$

Since we are interested now in $\widehat{\mathrm{S}}$, we can simplify the differential equation:

$$
s_{3} q \frac{d}{d q} \widehat{\mathrm{S}}=\left(s_{1}+s_{2}\right) \widehat{\mathrm{A}}+[\mathrm{B}, \widehat{\mathrm{S}}] \quad \bmod \left(s_{1}+s_{2}\right)^{2} .
$$

for

$$
\widehat{\mathrm{A}}=\left(\sum_{k>0} k \frac{(-q)^{k}}{(-q)^{k}-1} \alpha_{-k} \alpha_{k}\right) \circ \mathrm{S}(0)
$$

\footnotetext{
${ }^{7}$ The operator $\mathrm{M}$ was found earlier in the quantum cohomology of the Hilbert scheme of points of $\mathbb{C}^{2}$ [17. A parallel occurance appears in the local GromovWitten theory of curves 3 .
} 
DESCENDENTS ON LOCAL CURVES: STATIONARY THEORY 21

The eigenvectors for $\mathrm{B}\left(\bmod s_{1}+s_{2}\right)$ are the classes $\mathrm{P}_{\lambda}$ with eigenvalues

$$
w_{\lambda}=\sum_{(i, j) \in \lambda}(i-j) s_{1} .
$$

Equation (24) then gives a simple relationship between the entries of $\widehat{S}$ and of $\widehat{A}$ in the $P_{\lambda}$ basis.

The operator $\widehat{A}$ is diagonal in the Nakajima basis $C_{\lambda}$ with entries

$$
\widehat{\mathrm{A}}_{\lambda \lambda}^{\mathrm{C}}=\sum_{k \text { part of } \lambda} k^{2} \frac{(-q)^{k}}{(-q)^{k}-1} .
$$

Applying the change of basis formula (21), we obtain the entries in the $\mathrm{P}_{\lambda}$ basis $\left(\bmod s_{1}+s_{2}\right)$ :

$$
\widehat{\mathrm{A}}_{\mu^{\prime} \mu}^{\mathrm{P}}=\sum_{\lambda} \frac{\operatorname{dim} \mu^{\prime}}{\operatorname{dim} \mu} \frac{\chi^{\mu}(\lambda) \chi^{\mu^{\prime}(\lambda)}}{z(\lambda)} \sum_{k \text { part of } \lambda} k^{2} \frac{(-q)^{k}}{(-q)^{k}-1} .
$$

If we use the notation $\lambda_{r}$ for the number of parts in a partition $\lambda$ of size $r$, then we can rewrite the entries as:

$$
\widehat{\mathrm{A}}_{\mu^{\prime} \mu}^{\mathrm{P}}=\frac{\operatorname{dim} \mu^{\prime}}{\operatorname{dim} \mu} \sum_{r=1}^{d} r \frac{(-q)^{r}}{(-q)^{r}-1} \sum_{\lambda} \frac{\chi^{\mu}(\lambda) \chi^{\mu^{\prime}}(\lambda) r \lambda_{r}}{z(\lambda)} .
$$

The following Lemma (easily proven using the Murnaghan-Nakayama rule) gives a simpler expression for the innermost sum in the above expression.

Lemma 3. Let $\mu, \mu^{\prime}$ be partitions of the same size and let $r>0$. Then

$$
\sum_{\lambda} \frac{\chi^{\mu}(\lambda) \chi^{\mu^{\prime}}(\lambda) r \lambda_{r}}{z(\lambda)}=\sum_{\substack{\gamma, \gamma^{\prime} r-h o o k s \\ \mu \backslash \gamma=\mu^{\prime} \backslash \gamma^{\prime}}}(-1)^{h(\gamma)+h\left(\gamma^{\prime}\right)}
$$

where $h(\gamma)$ is the number of rows in a rim hook $\gamma$.

In the calculations below, we denote by $\theta_{r}\left(\mu, \mu^{\prime}\right)$ the quantity appearing in Lemma 3 ,

We now are able to compute the restriction

$$
\left.\frac{1}{s_{1}+s_{2}} \widehat{S}_{(d)}^{\mu}\right|_{s_{2}=-s_{1}}
$$

in terms of $\theta_{r}$ and the eigenvalues $w(\lambda)$ :

$$
\sum_{r=1}^{d}\left(\frac{(-1)^{d-1} s_{1}^{d-1}(d-1) !}{\operatorname{dim} \mu} \sum_{\nu} \chi^{\nu}((d)) \theta_{r}(\mu, \nu) \frac{r}{w(\mu)-w(\nu)+n_{\mu, \nu} s_{3}}\right) \frac{(-q)^{r}}{1-(-q)^{r}} .
$$


As before, $\chi^{\nu}((d))=0$ unless $\nu=\alpha_{a}$ is a hook. For $\theta_{r}(\mu, \nu)$ to be nonzero, we must have $\mu$ be the union of two hooks, of sizes $d-r$ and $r$. The integers $n_{\mu, \nu}$ which arise will not affect the answer.

If we multiply by the descendent and edge factors and take the constant term in $\frac{s_{3}}{s_{1}}$, we obtain an expression for (20) of the form

$$
\sum_{r=1}^{d} B_{r} \frac{(-q)^{r}}{1-(-q)^{r}}
$$

The explicit formulas for $B_{r}$ depend upon two cases. For $B_{d}$, we have

$$
\begin{aligned}
& (-1)^{d} d \cdot d !(d+2) ! B_{d}= \\
& \sum_{a+b=d-1}(-1)^{a}\left(\begin{array}{c}
d-1 \\
a
\end{array}\right) \sum_{\substack{i=-b \\
i \neq 0}}^{a} \frac{1}{i}\left(-(-b-1)^{d+2}+(-b)^{d+2}+a^{d+2}-(a+1)^{d+2}\right),
\end{aligned}
$$

and for $r<d$, we have

$$
\begin{aligned}
& \frac{(-1)^{d+r}}{2} d \cdot d !(d+2) ! B_{r}= \\
& \sum_{\substack{a+b=d-1 \\
a \geq r \\
0 \leq c \leq r-1}}(-1)^{a+c}\left(\begin{array}{c}
d-r-1 \\
a-r
\end{array}\right)\left(\begin{array}{c}
r-1 \\
c
\end{array}\right) \frac{(a-r-c)(b+c+1-r)}{(a-c)^{2}(b+c+1)} \\
& \quad \cdot\left(-(-b-1)^{d+2}+(-b)^{d+2}+(a-r)^{d+2}-(a-r+1)^{d+2}\right. \\
& \left.\quad-(c-r)^{d+2}+(c-r+1)^{d+2}+c^{d+2}-(c+1)^{d+2}\right) .
\end{aligned}
$$

4.5. Classical pairing. We compute the classical pairing $\left\langle\tau_{d}, \mathrm{C}_{(d[0])}\right\rangle$. The simpler pairing $\left\langle\tau_{d-1}, \mathrm{C}_{(d[0])}\right\rangle$ is needed for the calculation and is addressed first.

Lemma 4. $\left\langle\tau_{d-1}, \mathrm{C}_{(d[0])}\right\rangle=\frac{1}{d !}$

Proof. By dimension counting, the pairing has no dependence on $s_{1}$ and $s_{2}$, so we can work mod $s_{1}+s_{2}$. Localization then yields

$$
\begin{aligned}
& (-1)^{d} d \cdot d !(d+1) !\left\langle\tau_{d-1}, \mathrm{C}_{(d[0])}\right\rangle \\
& \quad=\sum_{a+b=d-1}(-1)^{a}\left(\begin{array}{c}
d-1 \\
a
\end{array}\right)\left(-(-b-1)^{d+1}+(-b)^{d+1}+a^{d+1}-(a+1)^{d+1}\right)
\end{aligned}
$$

If we rewrite $-(-b-1)^{d+1}+(-b)^{d+1}+a^{d+1}-(a+1)^{d+1}$ as a polynomial in $a$ alone, the leading term $-(d+1) d^{2} a^{d-1}$. Then,

$$
\left\langle\tau_{d-1}, \mathrm{C}_{(d[0])}\right\rangle=\frac{(-1)^{d}}{d \cdot d !(d+1) !}\left(-(d+1) d^{2}\right)(-1)^{d-1}(d-1) !=\frac{1}{d !}
$$


DESCENDENTS ON LOCAL CURVES: STATIONARY THEORY 23

since the contributions of all the lower terms are 0 .

We cannot compute $\left\langle\tau_{d}, \mathrm{C}_{(d[0])}\right\rangle$ in the same way, since we cannot work mod $s_{1}+s_{2}$ (as we know the answer is a multiple of $s_{1}+s_{2}$ ). Instead we work mod $s_{2}$ and consider the function

$$
f(k)=\left.(k+1) ! s_{1}^{d-1-k}\left\langle\tau_{k}, \mathbf{C}_{(d[0])}\right\rangle\right|_{s_{2}=0} .
$$

We can compute by localization that $f$ is of the form

$$
\sum_{i=1}^{d} c_{i} i^{k+1}
$$

for some constants $c_{i} \in \mathbb{Q}$. We also know

$$
f(0)=f(1)=\cdots=f(d-2)=0
$$

by dimension constraints. By Lemma 4, we have $f(d-1)=1$. Interpolation then gives $f(d)=\frac{d(d+1)}{2}$. We conclude the following result.

\section{Proposition 3.}

$$
\left\langle\tau_{d}, \mathrm{C}_{(d[0])}\right\rangle=\frac{s_{1}+s_{2}}{2 \cdot(d-1) !}
$$

\subsection{Proof of Theorem 3. We have}

$$
F(d)=F_{0}(d)+\sum_{r=1}^{d}\left(A_{r}+B_{r}\right) \frac{(-q)^{r}}{1-(-q)^{r}} .
$$

Although the formulas for $A_{r}$ and $B_{r}$ calculated in Sections 4.3 - 4.4 are very complicated, a wonderful combinatorial identity holds:

$$
A_{r}+B_{r}=\frac{1}{d !}
$$

for all $1 \leq r \leq d$. The proof of (25) is by straightforward manipulation using a few standard binomial sum identities. In the case $r=d$, all that is needed is the identity

$$
\sum_{i=0}^{m}(-1)^{i}\left(\begin{array}{c}
m \\
i
\end{array}\right)\left(a_{m} i^{m}+a_{m-1} i^{m-1}+\cdots+a_{0}\right)=(-1)^{m} m ! \cdot a_{m} .
$$

For $r<d$, the following two identities must also be used to compute the sum over $c$ in the expression for $B_{r}$ :

$$
\begin{gathered}
\sum_{i=0}^{m}(-1)^{i}\left(\begin{array}{c}
m \\
i
\end{array}\right) \frac{1}{x+i}=\frac{1}{(m+1)\left(\begin{array}{c}
x+m \\
m+1
\end{array}\right)} \\
\sum_{i=0}^{m}(-1)^{i}\left(\begin{array}{c}
m \\
i
\end{array}\right) \frac{1}{(x+i)^{2}}=\frac{1}{(m+1)\left(\begin{array}{c}
x+m \\
m+1
\end{array}\right)} \sum_{i=0}^{m} \frac{1}{x+i} .
\end{gathered}
$$


Combined with Proposition 3, the identity (25) yields

$$
F(d)=\frac{1}{2 \cdot d !} \sum_{r=1}^{d} \frac{1+(-q)^{r}}{1-(-q)^{r}}
$$

which completes the proof of Theorem 3 ,

The same method of computation actually yields a relatively simple formula for a larger family of invariants. Suppose that $m_{1}, \ldots, m_{k}$ are positive integers satisfying

$$
\sum_{i=1}^{k} m_{i}=d
$$

Lemma 2 relied only on a dimension analysis which also applies to $\mathbf{Z}_{d,(d)}^{\text {cap }}\left(\tau_{m_{1}}([0]) \cdots \tau_{m_{k}}([0])\right)^{\mathbf{T}}$, so we can expect the series to be relatively simple. In fact, we can prove

$$
\begin{aligned}
& \mathrm{Z}_{d,(d)}^{\mathrm{cap}}\left(\tau_{m_{1}}([0]) \cdots \tau_{m_{k}}([0])\right)^{\mathbf{T}}= \\
& \quad \frac{q^{d}}{m_{1} ! \cdots m_{k} !}\left(\frac{s_{1}+s_{2}}{s_{1} s_{2}}\right) \frac{1}{2} \sum_{r=1}^{d} C_{r}\left(m_{1}, \ldots, m_{k}\right) \frac{1+(-q)^{r}}{1-(-q)^{r}}
\end{aligned}
$$

for coefficients

$$
C_{r}\left(m_{1}, \ldots, m_{k}\right)=\sum_{\substack{I \subset\{1, \ldots, k\} \\ \sum_{i \in I} m_{i}<r}} r^{|I|-1}(d-r)^{k-|I|-1}\left(r-\sum_{i \in I} m_{i}\right) .
$$

For example, we have

$$
\begin{aligned}
& \mathrm{Z}_{3,(3)}^{\text {cap }}\left(\tau_{1}([0]) \tau_{2}([0])\right)^{\mathbf{T}}= \\
& \quad \frac{q^{3}}{2}\left(\frac{s_{1}+s_{2}}{s_{1} s_{2}}\right) \frac{1}{2}\left(2 \cdot \frac{1-q}{1+q}+2 \cdot \frac{1+q^{2}}{1-q^{2}}+3 \cdot \frac{1-q^{3}}{1+q^{3}}\right) .
\end{aligned}
$$

When proving (26) by the method used for Theorem 3, the leading classical term requires the somewhat surprising identity

$$
\sum_{r=1}^{d} C_{r}\left(m_{1}, \ldots, m_{k}\right)=\sum_{\substack{f:\{1, \ldots, k\} \rightarrow\{1, \ldots, k\} \\ f \text { has only one periodic orbit }}} \prod_{i=1}^{k} m_{f(i)} .
$$


DESCENDENTS ON LOCAL CURVES: STATIONARY THEORY 25

We can prove this identity by showing that both sides satisfy the same recurrence equation as a function of $m_{1}, \ldots, m_{k}$ :

$$
\begin{aligned}
F\left(m_{1}, \ldots, m_{k}\right)=m_{k}^{2}\left(m_{1}+\cdots+m_{k}\right)^{k-2} & +m_{1} F\left(m_{1}+m_{k}, m_{2}, \ldots, m_{k-1}\right) \\
& +m_{2} F\left(m_{1}, m_{2}+m_{k}, \ldots, m_{k-1}\right) \\
& +\cdots \\
& +m_{k-1} F\left(m_{1}, m_{2}, \ldots, m_{k-1}+m_{k}\right) .
\end{aligned}
$$

Finally, we can also use the same method of computation to obtain the analogous normalized Donaldson-Thomas partition functions. Surprisingly, these are equal to the stable pairs partition functions except in degree one:

$\mathrm{Z}_{d,(d)}^{\mathrm{DT}, \text { cap }}\left(\tau_{d}(\mathrm{p})\right)^{T}= \begin{cases}\mathrm{Z}_{d,(d)}^{\mathrm{cap}}\left(\tau_{d}(\mathrm{p})\right)^{T} & \text { if } d>1 \\ \mathrm{Z}_{d,(d)}^{\mathrm{cap}}\left(\tau_{d}(\mathrm{p})\right)^{T}+q\left(\frac{s_{1}+s_{2}}{s_{1} s_{2}}\right) q \frac{d}{d q} \log (M(-q)) & \text { if } d=1 .\end{cases}$

Here $M(q)=\prod\left(1-q^{r}\right)^{-r}$ is the MacMahon function.

\section{REFERENCES}

[1] K. Behrend, Donaldson-Thomas invariants via microlocal geometry, math.AG/0507523.

[2] T. Bridgeland, Hall algebras and curve-counting invariants, arXiv:1002.4372,

[3] J. Bryan and R. Pandharipande, The local Gromov-Witten theory of curves, JAMS 21 (2008), 101-136. math.AG/0411037.

[4] S. K. Donaldson and R. P. Thomas, Gauge theory in higher dimensions. In The geometric universe (Oxford, 1996), 31-47. Oxford Univ. Press, Oxford, 1998.

[5] C. Faber and R. Pandharipande, Hodge integrals and Gromov-Witten theory, Invent. Math. 139 (2000), 173-199.

[6] T. Graber and R. Pandharipande, Localization of virtual classes, Invent. Math., 135, 487-518, 1999. math.AG/9708001.

[7] D. Huybrechts and M. Lehn, The geometry of moduli spaces of shaves. Aspects of Mathematics, E31. Friedr. Vieweg \& Sohn, Braunschweig, 1997.

[8] D. Joyce and Y. Song, A theory of generalized Donaldson-Thomas invariants, arXiv:0810.5645.

[9] J. Le Potier, Systèmes cohérents et structures de niveau, Astérisque, 214, 1993.

[10] J. Li and B. Wu, Degeneration of Donaldson-Thomas invariants, preprint 2009.

[11] D. Maulik, A. Oblomkov, A. Okounkov, and R. Pandharipande, The Gromov-Witten/Donaldson-Thomas correspondence for toric 3-folds, Invent. Math. (to appear). arXiv:0809.3976. 
[12] D. Maulik, N. Nekrasov, A. Okounkov, and R. Pandharipande, GromovWitten theory and Donaldson-Thomas theory. I, Compos. Math. 142 (2006), 1263-1285.

[13] D. Maulik, N. Nekrasov, A. Okounkov, and R. Pandharipande, GromovWitten theory and Donaldson-Thomas theory. II, Compos. Math. 142 (2006), 1286-1304.

[14] D. Maulik and R. Pandharipande, A topological view of Gromov-Witten theory, Topology 45 (2006), 887-918.

[15] D. Maulik, R. Pandharipande, R. Thomas, Curves on K3 surfaces and modular forms, J. of Topology 3 (2010), 937-996. arXiv:1001.2719.

[16] A. Okounkov and R. Pandharipande, Virsoro constraints for target curves, Invent. Math. 163 (2006), 47-108.

[17] A. Okounkov and R. Pandharipande, The quantum cohomology of the Hilbert scheme of points of the plane, Invent. Math. 179 (2010), 523557.

[18] A. Okounkov and R. Pandharipande, The local Donaldson-Thomas theory of curves, Geom. Topol. 14 (2010), 1503-1567. math.AG/0512573.

[19] A. Okounkov and R. Pandharipande, The quantum differential equation of the Hilbert scheme of points of the plane, Transform. Groups $\mathbf{1 5}$ (2010), 965-982. arXiv/09063587.

[20] R. Pandharipande and A. Pixton, Descendents on local curves: rationality, arXiv:10114050.

[21] R. Pandharipande and A. Pixton, Descendent theory of stable pairs on toric 3-folds, arXiv:10114054.

[22] R. Pandharipande and R. P. Thomas, Curve counting via stable pairs in the derived category, Invent Math. 178 (2009), 407 - 447.

[23] R. Pandharipande and R. P. Thomas, The 3-fold vertex via stable pairs, Geom Topol. 13 (2009), 1835-1876.

[24] R. Pandharipande and R. P. Thomas, Stable pairs and BPS invariants, JAMS 23 (2010), 267-297.

[25] R. P. Thomas, A holomorphic Casson invariant for Calabi-Yau 3-folds, and bundles on K3 fibrations, J. Differential Geom. 54, 367-438, 2000.

[26] Y. Toda, Generating functions of stable pairs invariants via wallcrossings in derived categories, arXiv:0806.0062.

Departement Mathematik

ETH Zürich

rahul@math.ethz.ch

Department of Mathematics

Princeton University

apixton@math.princeton.edu
Department of Mathematics

Princeton University

rahulp@math.princeton.edu 